\title{
Percepção sobre uso de substâncias psicoativas em estudantes universitários: uma revisão integrativa
}

\author{
Emerson Feitosa Lins* \\ Kevan Guilherme Nóbrega Barbosa** \\ Mara Cristina Ribeiro***
}

\begin{abstract}
Resumo
A pesquisa buscou integrar o conhecimento relativo à percepção dos estudantes universitários em relação aos riscos no uso e abuso de substâncias psicoativas. Objetivou-se verificar se a percepção em relação ao risco poderia ser um fator que contribui para o uso, bem como a proximidade com os colegas e o pertencimento a grupos que façam uso dessas substâncias. Foram seguidos os passos de uma revisão integrativa. 27 estudos foram incluídos por estarem de acordo com os critérios estabelecidos. Os achados sugerem que há uma associação significativa entre a maneira como o estudante universitário percebe os riscos e o seu padrão de consumo de drogas, bem como a relação que ele estabelece com os pares e demais colegas. Outro aspecto relevante foi a evidência sobre o uso de substâncias que só podem ser adquiridas com prescrição médica e que são utilizadas de forma indiscriminada para o aumento do desempenho acadêmico.
\end{abstract}

Palavras-chave: Abuso de Substâncias Psicoativas, Universidade, Estudantes.

\section{Perception of psychoactive substance use in university students: an integrative review}

\begin{abstract}
The research aimed to integrate the knowledge related to the perception of university students regarding the risks in the use and abuse of psychoactive substances. The objective was to verify if the perception in relation to risk could be a factor that contributes to the use, as well as the proximity with colleagues and belonging to groups that make use of these substances. The steps of an integrative review were followed. 27 studies were included because they were in accordance with the established criteria. The findings suggest that there is a significant association between the way the university student perceives risks and his pattern of drug use, as well as the relationship he establishes with peers and other colleagues. Another relevant aspect was the evidence on the use of substances that can only be acquired with a medical prescription and that are used indiscriminately to increase academic performance.
\end{abstract}

Keywords: Psychoactive Substances Abuse, University, Students.

* Mestrando no Mestrado Profissional Pesquisa em Saúde do Centro Universitário CESMAC. Psicólogo no Instituto Federal de Alagoas.

** Doutorado em Odontologia pelo Programa de Pós-Graduação da Universidade Federal de Minas Gerais (Área de Concentração: Saúde Coletiva, Linha de Pesquisa: Epidemiologia e controle das doenças bucais). Professor do Centro Universitário CESMAC.

*** Doutorado em Ciências pela USP. Professora do Centro Universitário CESMAC e da Universidade Estadual de Ciência da Saúde de Alagoas. 


\section{Introdução}

Este artigo busca integrar o conhecimento relativo à percepção dos estudantes universitários em relação ao uso e abuso de substâncias psicoativas, considerando que estudos apontam que a experimentação de várias substâncias acaba fazendo parte da cultura universitária, onde os riscos existentes são ignorados (Willis, Adams \& Keene, 2019).

Historicamente, percebe-se que as drogas sempre se fizeram presente no cotidiano da humanidade, em diferentes culturas e na vida de pessoas de diferentes faixas etárias. Na contemporaneidade, não é diferente: independente do caráter lícito ou ilícito, o consumo permanece.

Para compreender melhor como o tema vem impactando a sociedade nos últimos anos, leva-se em conta o estudo feito pelo UNODC -Escritório das Nações Unidas sobre Drogas e Crime (2019) com aproximadamente 271 milhões de pessoas. Ou seja, 5,5\% da população mundial com idades compreendidas entre os 15 e os 64 anos para saber se elas haviam consumido drogas no ano anterior. Mesmo com o fato desses dados se assemelharem às estimativas de 2016, uma visão de longo prazo revela que o número de pessoas que consomem drogas é agora $30 \%$ superior ao de 2009 . Ainda que se leve em conta o aumento de $10 \%$ na população mundial de 15-64 anos, os dados mostram maior prevalência do consumo de opiáceos na África, Ásia, Europa e América do Norte e o consumo de cannabis na América do Norte, América do Sul e Ásia em comparação com 2009 (UNODC, 2019).

De modo semelhante às pesquisas realizadas com estudantes universitários de outras nacionalidades (Skidmore, Kaufman \& Crowell, 2016; Yi, Peltzer, Pengpid \& Susilowati, 2017), estudos demonstram que grande parte dos estudantes universitários brasileiros estão envolvidos no uso de múltiplas drogas e vulneráveis aos seus riscos (Oliveira, Alberghini, Santos \& Andrade, 2013). Nesse sentido, entende-se que há mudanças de vida importantes no momento de transição para a universidade que podem ser geradoras de estresse e causar impacto no bem-estar dos estudantes (Riordan \& Carey, 2019). Quando a qualidade de vida do indivíduo encontra-se comprometida, os domínios pessoais são afetados e, consequentemente, manifestam-se dificuldades em relacionamentos sociais e abuso de substâncias (Dyrbye, Massie, Eacker, Harper, Power, Durning, Thomas, Moutier, Satele, Sloan \& Shanafelt, 2010).

$\mathrm{O}$ ingresso em um ambiente acadêmico geralmente vem acompanhado de uma maior sensação de liberdade e ampliação das possibilidades de vida. Inserir-se em grupos e ter a sensação de pertencimento passa a ser algo buscado pelos estudantes. Ocorre, também, a necessidade de integração, aceitação e imitação dos comportamentos grupais (Rodríguez \& Scherer, 2008). Na universidade, a presença de acontecimentos e pessoas novas pode contribuir para o uso, além da rotina acadêmica composta por atividades avaliativas (Portugal \& Siqueira, 2011).

Frente a isto, estabeleceu-se como objetivo averiguar se a percepção dos estudantes universitários em relação ao risco poderia ser um fator que contribui para o uso. Um outro aspecto que foi investigado foi se a percepção e/ou o risco poderiam estar associados a outros fatores, como a proximidade com os colegas e o pertencimento a grupos que façam uso dessas substâncias. Os estudos citados foram realizados em diferentes países, o que amplia o escopo dessa revisão integrativa.

Ao pensar na relevância de explorar o tema, detecta-se que o entendimento de quais são as atitudes dos estudantes universitários em relação ao risco e à disponibilidade de substâncias contribui para a conscientização e iniciativas de prevenção no ambiente universitário (Lipari \& Jean-Francois, 2016).

\section{Metodologia}

Trata-se de artigo de revisão integrativa de literatura. A escolha se deu pelo fato de a revisão integrativa ser o tipo mais amplo de método de pesquisa do tipo revisão, permitindo a inclusão simultânea de pesquisas experimentais e não-experimentais para compreender melhor determinado fenômeno (Whittemore \& Knafl, 2005).

Como uma maneira de elaboração da revisão integrativa, foram seguidos os seguintes passos (Whittemore \& Knafl, 2005): identificação do problema, pesquisa na literatura, avaliação dos dados coletados, análise dos dados coletados e apresentação. 
A identificação do problema de pesquisa veio a partir de leituras conectadas com o tema, especificamente abordando questões relativas às atitudes frente a pessoas que fazem uso abusivo de drogas (Bakari \& Mann, 2019), o conhecimento das consequências e o uso de drogas em estudantes universitários (Williams \& Brands, 2019) e associação de pares e percepção dos riscos relacionados ao uso de maconha em adolescentes (Wynter \& Hynes, 2019).

Uma vez estabelecido o tema a ser pesquisado, o principal mecanismo utilizado para busca foi o site PubMed, pela sua relevância na área de Medicina e da Saúde.

O algoritmo utilizado para a busca foi baseado na estratégia PICo, onde P consiste no problema de pesquisa (drug use OR drug abuse), I consiste no interesse (risk and perception) e o Co consiste no contexto (students AND college OR university, NOT high school). Aqui, houve uma preocupação em restringir o público a ser pesquisado somente aos estudantes universitários, visto que estudantes de nível médio (high school) se encontram inseridos em um contexto que foge ao escopo da pesquisa. Dessa forma, o algoritmo de busca adotado foi:((drug abuse) or (drug use)) AND (risk) AND (perception) AND (students) AND ((college OR university) NOT high school)).

Objetivando refinar a pesquisa, foram utilizados os seguintes filtros: publicações nos últimos 5 anos e incluídas no MEDLINE.

Para avaliação dos estudos, foram considerados critérios de inclusão: estudos publicados na base de dados citada, dentro do intervalo de tempo de 2015 a 2020, no idioma inglês, cujo título fosse pertinente ao tema. Como critérios de exclusão, foram deixados de fora estudos que não estivessem voltados especificamente aos estudantes universitários. Uma vez feita a busca, os títulos e resumos foram lidos de forma crítica e os critérios de inclusão e exclusão foram aplicados, restando 27 artigos que foram lidos por completo.

Como forma de proceder à análise dos dados coletados, a leitura integral destes teve como finalidade apreender quais os aspectos consensuais sobre o tema, principalmente através de dados obtidos quantitativos nas pesquisas. Para melhor integração dos dados, os artigos foram aglomerados no Quadro I, com os objetivos de cada um suficientemente explícito para análise posterior.

\section{Resultados}

Após esses passos, restaram 111 artigos, dos quais 27 foram escolhidos por sua relevância de acordo com o tema escolhido.

Dos 27 artigos (100\%), 1 artigo (3,7\%) é do ano de 2020, 1 artigo (3,7\%) de 2019, 14 artigos (52\%) são de 2018, 3 artigos (11\%) são de 2017, 7 artigos (26\%) são de 2016, 1 artigos (3,7\%) é de 2015.

Quanto ao país de origem dos estudos, 20 (74\%) são dos Estados Unidos, 2 (7,4\%) do Brasil, 2 (7,4\%) do Canadá, 1 (3,7\%) da Colômbia, 1 (3,7\%) da Etiópia. 1 artigo $(3,7 \%)$ consiste em uma pesquisa que foi desenvolvida em 7 países da Europa.

Quanto ao tipo de estudo, 22 (81,5\%) são quantitativos, $3(11 \%)$ são qualitativos, 1 é revisão sistemática (3,7\%), 1 (3,7\%) é uma revisão de literatura.

Diante da análise dos dados encontrados nos estudos selecionados, os 3 principais temas identificados foram relacionados às atitudes dos estudantes diante do uso/abuso de substâncias, cujo detalhamento se deu através da análise dos seguintes itens:

a) A influência do uso dos pares/colegas no uso pessoal;

b) A percepção e o conhecimento em relação aos riscos e como isso pode influenciar o uso pessoal;

c) Possibilidades interventivas como forma de prevenção e/ou redução do uso.

\section{Discussão}

Atitudes dos estudantes diante do uso/ abuso de substâncias

\section{a) A influência do uso dos pares/colegas no uso pessoal}

Ao se debruçar sobre a temática do uso e abuso de substâncias no que concerne aos estudantes universitários, verificou-se que há indícios de que os estudantes sejam influenciados a fazer uso de substâncias levando em consideração muito mais os benefícios do que os riscos (Galván, Sánchez-Carballo, Gómez-Morales, Humánez-Julio, Guerrero-Martelo \& Vásquez De la Hoz, 2016), como no 


\section{Quadro 1 - Agrupamento dos artigos utilizados na revisão integrativa}

\begin{tabular}{|c|c|c|}
\hline $\begin{array}{l}\text { Autores do artigo e ano de } \\
\text { publicação }\end{array}$ & Tipo de estudo & Objetivo do estudo \\
\hline $\begin{array}{l}\text { Kollath-Cattano, Hatteberg e } \\
\text { Kooper (2020) }\end{array}$ & Quantitativo & $\begin{array}{l}\text { Examinar a prevalência e correlatos do uso de substâncias ilícitas } \\
\text { por estudantes universitários e identificar como diferentes per- } \\
\text { cepções relacionadas às drogas estão relacionadas ao uso dessas } \\
\text { substâncias. }\end{array}$ \\
\hline Willis, Adams e Keene (2019) & Qualitativo & $\begin{array}{l}\text { Compreender como os estudantes desenvolvem atitudes sobre os } \\
\text { possíveis riscos e recompensas em comportamentos como beber } \\
\text { imoderadamente e uso de drogas. }\end{array}$ \\
\hline $\begin{array}{l}\text { Acuff, Soltis, Luciano, Me- } \\
\text { shesha, Pedrelli, Dennhardt e } \\
\text { Murphy (2018) }\end{array}$ & Quantitativo & $\begin{array}{l}\text { Examinar sintomas depressivos como um preditor prospectivo de } \\
\text { mudanças relacionadas ao consumo de álcool em estudantes univer- } \\
\text { sitários que fazem uso abusivo. }\end{array}$ \\
\hline $\begin{array}{l}\text { Balthazar, Gaino, Almeida, } \\
\text { Oliveira e Souza (2018) }\end{array}$ & Qualitativo & $\begin{array}{l}\text { Analisar a percepção dos líderes estudantis da graduação em enfer- } \\
\text { magem sobre as motivações e/ou fatores de risco para o consumo } \\
\text { de substâncias no ambiente universitário. }\end{array}$ \\
\hline $\begin{array}{l}\text { Dillard, Ferrer, Bulthuis e } \\
\text { Klein (2018) }\end{array}$ & Quantitativo & $\begin{array}{l}\text { Examinar se os protótipos de estudantes universitários de um indi- } \\
\text { víduo que frequentemente bebe "além do limite" estavam associa- } \\
\text { dos ao seu próprio consumo de álcool. }\end{array}$ \\
\hline $\begin{array}{l}\text { Fevrier, Nabors, Vidourek e } \\
\text { King (2018) }\end{array}$ & Quantitativo & $\begin{array}{l}\text { Identificar e descrever padrões/diferenças potenciais no uso de } \\
\text { narguilé por estudantes universitários, e as relações entre atitudes e } \\
\text { conhecimentos sobre o uso e uso dessa droga. }\end{array}$ \\
\hline $\begin{array}{l}\text { Gebremariam, Mruts e Ne- } \\
\text { way (2018) }\end{array}$ & Quantitativo & $\begin{array}{l}\text { Avaliar a prevalência e os preditores de substâncias entre estudantes } \\
\text { da Universidade Debre Berhan. }\end{array}$ \\
\hline $\begin{array}{l}\text { Halladay, Petker, Fein, Munn } \\
\text { e MacKillop (2018) }\end{array}$ & Revisão sistemática & $\begin{array}{l}\text { Investigar, nos jovens de } 15 \text { a } 24 \text { anos, qual o conteúdo e o efeito } \\
\text { de breves intervenções (1-2 sessões) para o uso da cannabis sobre } \\
\text { (a) resultados relacionados com a cannabis, (b) outros usos da subs- } \\
\text { tância, (c) comportamentos de busca de ajuda, (d) saúde mental e } \\
\text { bem-estar, e (e) resultados acadêmicos e profissionais. }\end{array}$ \\
\hline $\begin{array}{l}\text { Kenney, DiGuiseppi, Meisel, } \\
\text { Balestrieri e Barnett (2018) }\end{array}$ & Quantitativo & $\begin{array}{l}\text { Examinar relações transversais entre sintomas de ansiedade e de- } \\
\text { pressão, percepções descritivas e injuntivas de pares importantes, } \\
\text { consumo de álcool e problemas relacionados ao álcool em uma rede } \\
\text { social de estudantes universitários do primeiro semestre. }\end{array}$ \\
\hline $\begin{array}{l}\text { Keough, O’Connor e Stewart } \\
\text { (2018) }\end{array}$ & Quantitativo & $\begin{array}{l}\text { Investigar se o consumo frequente de álcool de forma solitária } \\
\text { entre adultos jovens é preditor de problemas graves de álcool que } \\
\text { atendem aos critérios diagnósticos para AUD (desordens causadas } \\
\text { pelo uso de álcool, sigla em inglês). }\end{array}$ \\
\hline $\begin{array}{l}\text { Krieger, Young, Anthenien e } \\
\text { Neighbors (2018) }\end{array}$ & Revisão de literatura & $\begin{array}{l}\text { Resumir as tendências e os fatores de desenvolvimento e sociais que } \\
\text { afetam a probabilidade, os fatores de risco e proteção relacionados } \\
\text { e as consequências negativas dos comportamentos de consumo } \\
\text { excessivo de álcool. }\end{array}$ \\
\hline
\end{tabular}




\begin{tabular}{|c|c|c|}
\hline $\begin{array}{l}\text { Autores do artigo e ano de } \\
\text { publicação }\end{array}$ & Tipo de estudo & Objetivo do estudo \\
\hline $\begin{array}{l}\text { Lehne, Zeeb, Pischke, Miko- } \\
\text { lajczyk, Bewick, McAlaney, } \\
\text { Dempsey, Van Hal, Stock, } \\
\text { Akvardar, Kalina, Orosova, } \\
\text { Aguinaga-Ontoso, Guillen- } \\
\text {-Grima e Helmer (2018) }\end{array}$ & Quantitativo & $\begin{array}{l}\text { Investigar se as percepções e aprovação de uso pelos colegas estão } \\
\text { associadas ao à aprovação e uso pessoal de sedativos não prescritos } \\
\text { e pílulas para dormir. }\end{array}$ \\
\hline $\begin{array}{l}\text { Ross, Arria, Brown, Mullins, } \\
\text { Schiffman, Simoni-Wastila, } \\
\text { dosReis (2018) }\end{array}$ & Quantitativo & $\begin{array}{l}\text { Examinar a variação em estudantes universitários dos riscos e bene- } \\
\text { fícios percebidos em relação ao uso dos NPS (uso não-médico de } \\
\text { estimulantes prescritos, sigla em inglês). }\end{array}$ \\
\hline $\begin{array}{l}\text { Salloum, Krauss, Agrawal, } \\
\text { Bierut e Grucza (2018) }\end{array}$ & Quantitativo & $\begin{array}{l}\text { Quantificar as associações recíprocas entre o uso de cannabis e } \\
\text { percepção de risco em adultos jovens. }\end{array}$ \\
\hline $\begin{array}{l}\text { Buckner, Lemke e Walukevi- } \\
\text { ch (2017) }\end{array}$ & Quantitativo & $\begin{array}{l}\text { Investigar os fatores de vulnerabilidade psicológica para o suicídio } \\
\text { em usuários de cannabis. }\end{array}$ \\
\hline $\begin{array}{l}\text { Pearson, Bravo, Conner e } \\
\text { Team (2017) }\end{array}$ & Quantitativo & $\begin{array}{l}\text { Pesquisar sobre os efeitos dos padrões típicos de uso da maconha e } \\
\text { se existem subgrupos distintos de usuários de maconha em estudan- } \\
\text { tes universitários. }\end{array}$ \\
\hline $\begin{array}{l}\text { Galván, Sánchez-Carballo, } \\
\text { Gómez-Morales, Humánez- } \\
\text {-Julio, Guerrero-Martelo e } \\
\text { Vásquez De la Hoz (2016) }\end{array}$ & Quantitativo & $\begin{array}{l}\text { Avaliar e comparar as crenças sobre a cannabis, seu uso e poten- } \\
\text { ciais consequências entre dois grupos de estudantes universitários } \\
\text { colombianos. }\end{array}$ \\
\hline $\begin{array}{l}\text { LaBrie, Earle, Boyle, Hum- } \\
\text { mer, Montes, Turrisi, Napper } \\
(2016)\end{array}$ & Quantitativo & $\begin{array}{l}\text { Avaliar uma intervenção baseada em normas sociais para pais de } \\
\text { alunos do primeiro ano de faculdade. }\end{array}$ \\
\hline Lipari e Jean-Francois (2016) & Quantitativo & $\begin{array}{l}\text { Examinar as atitudes dos estudantes universitários sobre os riscos } \\
\text { associados ao uso de substâncias e sua percepção da disponibilidade } \\
\text { das substâncias. }\end{array}$ \\
\hline $\begin{array}{l}\text { Miller, DiBello, Lust, Carey e } \\
\text { Carey (2016) }\end{array}$ & Quantitativo & $\begin{array}{l}\text { Determinar se o sono adequado modera a associação prospectiva } \\
\text { entre a quantidade de bebida semanal e as consequências. }\end{array}$ \\
\hline $\begin{array}{l}\text { Nair, Nemeth, Sommers, } \\
\text { Newman e Amella (2016) }\end{array}$ & Qualitativo & $\begin{array}{l}\text { Examinar as percepções dos estudantes de enfermagem sobre os fa- } \\
\text { tores de risco e proteção associados aos comportamentos alcoólicos } \\
\text { entre eles e seus pares e identificar políticas de uso de substâncias e } \\
\text { sua influência. }\end{array}$ \\
\hline $\begin{array}{l}\text { Tuliao, Jaffe e McChargue } \\
\text { (2016) }\end{array}$ & Quantitativo & $\begin{array}{l}\text { Examinar várias expectativas de resultados do uso de álcool associa- } \\
\text { do ao Transtorno do Estresse Pós-Traumático (TEPT). }\end{array}$ \\
\hline $\begin{array}{l}\text { Kilmer, Geisner, Gasser e } \\
\text { Lindgren (2015) }\end{array}$ & Quantitativo & $\begin{array}{l}\text { Examinar as percepções normativas e fatores demográficos a estas } \\
\text { associados em estudantes universitários em relação ao uso não- } \\
\text {-médico de estimulantes prescritos. }\end{array}$ \\
\hline
\end{tabular}


caso do uso de estimulantes com a finalidade de melhorar o desempenho acadêmico (Ross, Arria, Brown, Mullins, Schiffman, Simoni-Wastila \& dosReis, 2018). Quando os estudantes percebem que o uso de estimulantes é uma norma entre seus pares, isso pode representar um fator de risco para a iniciação ou a manutenção do uso (Kilmer, Geisner, Gasser \& Lindgren, 2015).

Quando a percepção relativa ao risco é baixa, isso pode ser associado ao consumo, como no caso dos cigarros eletrônicos (Cooper, Loukas, Case, Marti \& Perry, 2018) e da maconha (Halladay, Petker, Fein, Munn \& MacKillop, 2018; Salloum, Krauss, Agrawal, Bierut \& Grucza, 2018). Essa percepção de baixo risco pode representar, inclusive, um obstáculo à procura pelo tratamento adequado para o uso abusivo de substâncias (Halladay, Petker, Fein, Munn \& MacKillop, 2018). É relevante observar que a literatura destaca o fato de os usuários de maconha serem um grupo heterogêneo de pessoas com diferentes perfis de fatores de risco/proteção (Pearson, Bravo \& Conner, 2017).

Além disso, estudos apontam que é possível encontrar taxas mais elevadas de consumo excessivo de álcool e problemas correlatos entre aqueles que são colegas de faculdade do que entre aquele que não o são (Krieger, Young, Anthenien \& Neighbors, 2018). A influência dos amigos, a curiosidade e a busca de diversão são condições que facilitam o uso de substâncias (Balthazar, Gaino, Almeida, Oliveira \& Souza, 2018). A depender de quais fatores se combinam, a influência dos colegas pode ser tanto um fator de proteção quanto um fator de risco (Nair, Nemeth, Sommers, Newman \& Amella (2016). Isso mostra que a questão das relações humanas e a necessidade de pertencimento a grupos são quesitos a serem levados em consideração diante de uma problematização do tema.

\section{b) A percepção e o conhecimento em relação} aos riscos e como isso pode influenciar o uso pessoal

$\mathrm{Na}$ realidade brasileira, em uma pesquisa que comparou estudantes que não tinham o hábito de beber excessivamente com aqueles que o tinham, estes apresentaram maiores escores em testes que identificam transtornos relacionados ao álcool, além de apresentarem percepção reduzida a respeito da negatividade das consequências, apesar destas estarem presentes (Bedendo, Andrade, Opaleye \& Noto, 2017). De modo semelhante, há estudos que evidenciam a existência de uma percepção, por parte dos estudantes universitários, de baixo risco em relação ao hábito de fumar narguilé e apontam para a importân- cia da prevenção ao uso (Fevrier, Nabors, Vidourek \& King, 2018).

No que diz respeito aos hábitos de vida dos estudantes universitários, estudos apontam que há uma associação entre privação do sono e uso excessivo de álcool (Miller, DiBello, Lust, Carey \& Carey, 2016). Somando-se a essa informação, pesquisas demonstram que o uso de sedativos e pílulas para dormir sem prescrição médica por parte dos estudantes universitários tornou-se um problema de saúde pública, havendo uma correlação entre ter uma percepção de que os colegas fazem uso abusivo e ter a tendência alta de usá-los ao longo da vida (Lehne, Zeeb, Pischke, Mikolajczyk, Bewick, McAlaney, Dempsey, Van Hal, Stock, Akvardar, Kalina, Orosova, Aguinaga-Ontoso, Guillen-Grima \& Helmer, 2018). Destaca-se, ainda, o hábito de beber de forma solitária. Embora seja incomum em adultos jovens, ao se fazer presente pode indicar uma perda de controle, levando ao uso abusivo e problemas posteriores (Keough, O'Connor \& Stewart, 2018).

No que tange à saúde mental, os problemas atrelados ao uso abusivo de álcool podem ser exacerbados por sintomas depressivos, que são comuns entre estudantes universitários (Acuff, Soltis, Luciano, Meshesha, Pedrelli, Dennhardt \& Murphy, 2018). No contexto universitário, o álcool por vezes é utilizado para lidar com as dificuldades decorrentes do transtorno de estresse pós-traumático (Tuliao, Jaffe \& McChargue, 2016). Um outro aspecto relevante a ser citado em relação ao aspecto psicológico é a vulnerabilidade existente nos usuários de cannabis em relação ao risco de suicídio (Buckner, Lemke \& Walukevich, 2017).

c) Possibilidades interventivas como forma de prevenção e/ou redução de uso

Dentre diversos fatores, destacam-se alguns específicos na literatura que interferem no comportamento de uso de substâncias: ser do sexo masculino (Gebremariam, Mruts \& Neway, 2018) e ter familiares e amigos que fazem uso de substâncias (Gebremariam, Mruts \& Neway, 2018; Kenney, DiGuiseppi, Meisel, Balestrieri \& Barnett, 2018). No ambiente universitário, quando o(a) estudante percebe que os seus colegas mais próximos bebem de forma abusiva, isso pode predizer o seu próprio comportamento em relação à ingestão de álcool (Dillard, Ferrer, Bulthuis \& Klein, 2018; Kenney, DiGuiseppi, Meisel, Balestrieri \& Barnett, 2018).

Nesse sentido, convém pensar como o uso de substâncias por parte dos homens vem a reforçar os estereótipos de gênero e reafirmação da masculinidade, ainda 
que possam produzir danos à saúde. Uma possibilidade de intervenção é a adoção de políticas públicas voltadas especificamente para esse público, visto que ser do sexo masculino é um fator associado à uma maior prevalência de uso de substâncias (Gebremariam, Mruts \& Neway, 2018).

Quantos aos estudantes universitários, pesquisas indicam que realizar uma intervenção com os pais de alunos que estão iniciando na universidade, ainda no primeiro ano, pode contribuir para a redução do uso de álcool, especialmente o uso excessivo (LaBrie, Earle, Boyle, Hummer, Montes, Turrisi \& Napper, 2016). Assim, pode-se conceber o desenvolvimento de ideias semelhantes, envolvendo não apenas o aluno, mas também as pessoas mais significativas que o cercam, para uma maior efetividade e abrangência ações preventivas.

Estudos frisam que a prevenção pode ser direcionada, inclusive, para aqueles estudantes que já fazem uso de maconha (Holt \& Looby, 2018; Kollath-Cattano, Hatteberg \& Kooper, 2020), por entender que estes possuem maior risco de utilizar outras substâncias ilícitas (Kollath-Cattano, Hatteberg \& Kooper, 2020). Essa é uma preocupação pois considera-se que a percepção da maconha como algo prejudicial vem declinando desde 1991 nos Estados Unidos (Keyes, Wall, Cerdá, Schulenberg, O’Malley, Galea, Feng \& Hasin, 2016).

\section{Considerações Finais}

O contato com a literatura especializada traz à luz a relação direta entre a maneira como os estudantes universitários percebem o risco em relação ao uso de substâncias e a tendência ao uso. Os achados sugerem que há uma associação significativa entre a maneira como o estudante universitário percebe os riscos e o seu padrão de consumo de drogas, bem como a relação que ele estabelece com os pares e demais colegas. Um outro aspecto relevante do artigo é evidenciar a questão do uso de substâncias que só podem ser utilizadas com prescrição médica e que são utilizadas de forma indiscriminada para aumento do desempenho acadêmico, especialmente em ambientes competitivos. A associação existente entre o uso da maconha e o risco de suicídio é especialmente preocupante; pensando nesse sentido, pode-se ampliar espaços onde o tema possa ser discutido e analisado, considerando-o como um fenômeno multideterminado e que possui implicações múltiplas, além de realizar mais estudos para averiguação a respeito do tema em outros contextos.

Há necessidade de continuar realizando trabalhos futuros, pesquisando o tema em diferentes realidades, con- siderando as especificidades de cada país, principalmente pelo debate que tem sido trazido constantemente em diferentes veículos de comunicação sobre os processos de descriminalização do uso da maconha, enquanto continua existindo a ênfase para o uso do álcool de forma recreativa e significativamente integrada na convivência social, especialmente na universidade. Em termos de aplicações práticas, tais pesquisas poderão contribuir para a criação de medidas preventivas mais eficazes e o aperfeiçoamento daquelas que já existem no âmbito acadêmico.

\section{Referências}

Acuff, S. F., Soltis, K. E., Luciano, M. T., Meshesha, L. Z., Pedrelli, P., Dennhardt, A. A., \& Murphy, J. G. (2018). Depressive symptoms as predictors of alcohol problem domains and reinforcement among heavy drinking college students. Psychol Addict Behav, 32(7), 792-799. https://doi. org/10.1037/adb0000397

Bakari, I., \& Mann, R. (2019). Attitudes toward persons who abuse drugs in one urban community of woodbrook in port of Spain-Trinidad \& Tobago. Texto Contexto Enferm, 28(Spe), e914. https://doi.org/10.1590/1980-265X-TCE-CICAD-9-14

Balthazar, E. B., Gaino, L. V., Almeida, L. Y. de, Oliveira, J. L. de, \& Souza, J. de. (2018). Risk factors for substance use: perception of student leaders Rev Bras Enferm, 71(Suppl 5), 2116-2122. https://doi.org/10.1590/00347167-2017-0587

Bedendo, A., Andrade, A. L. M., Opaleye, E. S., \& Noto, A. R. (2017). Binge drinking: a pattern associated with a risk of problems of alcohol use among university students. Rev Latino-Am Enfermagem, 25, e2925. https:/ / doi.org/10.1590/1518-8345.1891.2925

Buckner, J. D., Lemke, A. W., \& Walukevich, K. A. (2017). Cannabis use and suicidal ideation: Test of the utility of the interpersonal-psychological theory of suicide. Psychiatry Res, 253, 256-259. https://doi.org/10.1016/j. psychres.2017.04.001

Cooper, M., Loukas, A., Case, K. R., Marti, C. N., \& Perry, C. L. (2018). A longitudinal study of risk perceptions and e-cigarette initiation among college students: Interactions with smoking status. Drug Alcohol Depend, 186, 257-263. https://doi.org/10.1016/j.drugalcdep.2017.11.027

Dillard, A. J., Ferrer, R. A., Bulthuis, K. R. K., \& Klein, W. M. P. (2018). Positive excessive drinker prototypes predict greater drinking and alcohol problems. British Journal of Health Psychology, 23(4), 1000-1020. https://doi. org/10.1111/bjhp.12335

Dyrbye, L. N., Massie, F. S., Eacker, A., Harper, W., Power, D., Durning, S. J. Thomas, M. R., Moutier, C., Satele, D., Sloan, J., \& Shanafelt, T. D. (2010). Relationship Between Burnout and Professional Conduct and Attitudes Among US Medical Students. JAMA, 304(11), 1173-1180. https://doi. org/10.1001/jama.2010.1318

Fevrier, B., Nabors, L., Vidourek, R. A., \& King, K. A. (2018). Hookah Use Among College Students: Recent Use, Knowledge of Health Risks, Attitude and Reasons for Use. J Community Health, 43(6), 1037-1043. https://doi. org/10.1007/s10900-018-0519-8

Galván, G., Sánchez-Carballo, Á., Gómez-Morales, I., Humánez-Julio, O., Guerrero-Martelo, M., \& Vásquez De la Hoz, F. (2016). Belief system regarding Cannabis, its use and consequences: Users versus non-users in colombian university students. Vertex, XXVII(130), 434-442. https:// pubmed.ncbi.nlm.nih.gov/28898302

Gebremariam, T. B., Mruts, K. B., \& Neway, T. K. (2018). Substance use and associated factors among Debre Berhan University students, Central Ethiopia. Subst Abuse Treat Prev Policy, 13(1), 13. https://doi.org/10.1186/ s13011-018-0150-9

Halladay, J., Petker, T., Fein, A., Munn, C., \& MacKillop, J. (2018). Brief interventions for cannabis use in emerging adults: protocol for a systematic review, meta-analysis, and evidence map. Syst Rev, 7(1), 106. https://doi. org/10.1186/s13643-018-0772-z

Holt, L. J., \& Looby, A. (2018). Factors that Differentiate Prescription Stimulant 
Misusers from those At-Risk for Misuse: Expectancies, Perceived Safety, and Diversion. Subst Use Misuse, 53(7), 1068-1075. https://doi.org/10.10 80/10826084.2017.1392984

Kenney, S. R., DiGuiseppi, G. T., Meisel, M. K., Balestrieri, S. G., \& Barnett, N. P. (2018). Poor mental health, peer drinking norms, and alcohol risk in a social network of first-year college students. Addict Behav, 84, 151-159. https://doi.org/10.1016/j.addbeh.2018.04.012

Keough, M. T., O’Connor, R. M., \& Stewart, S. H. (2018). Solitary drinking is associated with specific alcohol problems in emerging adults. Addict Bebav, 76, 285-290. https://doi.org/10.1016/j.addbeh.2017.08.024

Keyes, K. M., Wall, M., Cerdá, M., Schulenberg, J., O’Malley, P. M., Galea, S., Feng, T., \& Hasin, D. S. (2016). How does state marijuana policy affect US youth? Medical marijuana laws, marijuana use and perceived harmfulness: 1991-2014. Addiction, 111(12), 2187-2195. https://doi.org/10.1111/ add. 13523

Kilmer, J. R., Geisner, I. M., Gasser, M. L., \& Lindgren, K. P. (2015). Normative perceptions of non-medical stimulant use: Associations with actual use and hazardous drinking. Addict Behav, 111(12), 2187-2195. https://doi. org/10.1016/j.addbeh.2014.11.005

Kollath-Cattano, C., Hatteberg, S. J., \& Kooper, A. (2020). Illicit drug use among college students: The role of social norms and risk perceptions. Addict Behav, 105, 106289. https://doi.org/10.1016/j.addbeh.2020.106289

Krieger, H., Young, C. M., Anthenien, A. M., \& Neighbors, C. (2018). The Epidemiology of Binge Drinking Among College-Age Individuals in the United States. Alcobol Res, 39(1), 23-30.

LaBrie, J. W., Earle, A. M., Boyle, S. C., Hummer, J. F., Montes, K., Turrisi, R., \& Napper, L. E. (2016). A parent-based intervention reduces heavy episodic drinking among first-year college students. Psychol Addict Behav, 30(5), 523-535. https://doi.org/10.1037/adb0000187

Lehne, G., Zeeb, H., Pischke, C. R., Mikolajczyk, R., Bewick, B. M., McAlaney, J., Dempsey, R. C., Van Hal, G., Stock, C., Akvardar, Y., Kalina, O., Orosova, O., Aguinaga-Ontoso, I., Guillen-Grima, F., \& Helmer, S. M. (2018). Personal and perceived peer use and attitudes towards use of non-prescribed prescription sedatives and sleeping pills among university students in seven European countries. Addict Behav, 87, 17-23. https:// doi.org/10.1016/j.addbeh.2018.06.012

Lipari, R., \& Jean-Francois, B. (2016). Trends in Perception of Risk and Availability of Substance Use Among Full-Time College Students. In The CBHSQ Report (pp. 1-12). Substance Abuse and Mental Health Services Administration (US). http://www.ncbi.nlm.nih.gov/pubmed/27854410

Miller, M. B., DiBello, A. M., Lust, S. A., Carey, M. P., \& Carey, K. B. (2016). Adequate sleep moderates the prospective association between alcohol use and consequences. Addict Behav, 63, 23-28. https://doi.org/10.1016/j. addbeh.2016.05.005

Nair, J. M., Nemeth, L. S., Sommers, M., Newman, S., \& Amella, E. (2016). Alcohol Use, Misuse, and Abuse Among Nursing Students. J Addict Nurs, 27(1), 12-23. https://doi.org/10.1097/JAN.0000000000000107

Oliveira, L. G. de, Alberghini, D. G., Santos, B. dos, \& Andrade, A. G. de. (2013). Polydrug use among college students in Brazil: a nationwide survey. Rev Bras Psiquitr, 35(3), 221-230. https://doi.org/10.1590/15164446-2012-0775

Pearson, M. R., Bravo, A. J., \& Conner, B. T. (2017). Distinguishing subpopulations of marijuana users with latent profile analysis. Drug Alcohol Depend, 172, 1-8. https://doi.org/10.1016/j.drugalcdep.2016.10.043
Portugal, F. B., \& Siqueira, M. M. de. (2011). Fatores associados ao uso de substâncias psicoativas entre universitários de pedagogia da Universidade Federal do Espírito Santo. Cad Saúde Colet, 19(3), 348-355. http://www.cadernos. iesc.ufrj.br/cadernos/images/csc/2011_3/artigos/csc_v19n3_348-355.pdf

Riordan, B. C., \& Carey, K. B. (2019). Wonderland and the rabbit hole: A commentary on university students' alcohol use during first year and the early transition to university. Drug Alcobol Rev, 38(1), 34-41. https://doi. $\operatorname{org} / 10.1111 /$ dar.12877

Rodríguez, V. M. H., \& Scherer, Z. A. P. (2008). Motivação do estudante universitário para o consumo de drogas legais. Rev Latino-Am Enfermagem, 16(Especial), 6.

Ross, M. M., Arria, A. M., Brown, J. P., Mullins, C. D., Schiffman, J., Simoni-Wastila, L., \& DosReis, S. (2018). College students' perceived benefit-to-risk tradeoffs for nonmedical use of prescription stimulants: Implications for intervention designs. Addict Behav, 79, 45-51. https://doi.org/10.1016/j. addbeh.2017.12.002

Salloum, N. C., Krauss, M. J., Agrawal, A., Bierut, L. J., \& Grucza, R. A. (2018). A reciprocal effects analysis of cannabis use and perceptions of risk. Addiction, 113(6), 1077-1085. https://doi.org/10.1111/add.14174

Skidmore, C. R., Kaufman, E. A., \& Crowell, S. E. (2016). Substance Use Among College Students. Child Adolesc Psychiatric Clin N Am, 25(4), 735-753. https://doi.org/10.1016/j.chc.2016.06.004

Tuliao, A. P., Jaffe, A. E., \& McChargue, D. E. (2016). Alcohol Expectancies, Posttraumatic Stress Disorder, and Alcohol Use in College Students With a History of Childhood Trauma. J Dual Diagn, 12(1), 4-14. https://doi.or $\mathrm{g} / 10.1080 / 15504263.2016 .1146382$

UNODC. (2019). Executive Summary, Conclusions And Policy Implications. In Word Drug Report - 2019 (pp. 1-66). United Nations publication. https:// doi.org/10.18356/bdc264f4-en

Whittemore, R., \& Knafl, K. (2005). The integrative review: updated methodology. J Adv Nurs, 52(5), 546-553. https://doi.org/10.1111/j.13652648.2005.03621.x

Williams, F., \& Brands, B. (2019). Knowledge of consequences, academic performance and drug consumption among undergraduate students in one university in Jamaica. Texto Contexto Enferm, 28(Spe), e213. https:// doi.org/10.1590/1980-265x-tce-cicad-2-13

Willis, E., Adams, R., \& Keene, J. (2019). If Everyone Is Doing It, It Must Be Safe: College Students' Development of Attitudes toward Poly-Substance Use. Subst Use Misuse, 54(11), 1886-1893. https://doi.org/10.1080/1082 6084.2019.1618334

Wynter, J., \& Hynes, M. (2019). Peer association and perception of harms and benefits associated marijuana use among Jamaican adolescents. Texto Contexto Enferm, 28(Spe), e187. https://doi.org/10.1590/1980-265x-tce-cicad-18-7

Yi, S., Peltzer, K., Pengpid, S., \& Susilowati, I. H. (2017). Prevalence and associated factors of illicit drug use among university students in the association of southeast Asian nations (ASEAN). Subst Abuse Treat Prev Policy, 12(1), 9. https://doi.org/10.1186/s13011-017-0096-3

Submetido em: 5-6-2020

Aceito em: 2-2-2021 